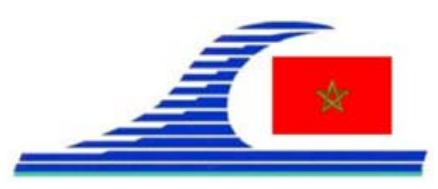

Conférence Méditerranéenne Côtière et Maritime EDITION 2, TANGER, MAROC (2011)

Coastal and Maritime Mediterranean Conference

Disponible en ligne - http://www.paralia.fr - Available online

\title{
Valorisation des sédiments de dragage portuaire du nord du Maroc dans des matériaux de construction en terre cuite
}

\author{
Laïla BEN ALLAL ${ }^{1}$, Mohammed AMMARI ${ }^{1}$, Amina AZMANI ${ }^{1}$, \\ Sanae LAMRANI ${ }^{1}$, Ikram FRAR ${ }^{1}$
}

\author{
1. Laboratoire de Génie Chimique et Valorisation des Ressources (LGCVR), \\ FST Tanger, Maroc. \\ l.benallal@fstt.ac.ma
}

\section{Résumé :}

L'objectif de ce travail est de valoriser les sédiments de dragage des ports de Tanger et de Larache dans des matériaux de construction en terre cuite et plus précisément dans les briques. L'étude a consisté à élaborer des échantillons de briques à base de sédiments à l'échelle du laboratoire. Le taux de substitution optimum d'argile entrant dans la formulation des briques par les sédiments a été évalué à l'aide d'essais mécaniques. Les paramètres tels que la surface spécifique des mélanges, la porosité, l'absorption d'eau et la masse volumique apparente influençant le comportement des briques ont été déterminés.

\section{Mots clés :}

Briques - Valorisation - Sédiments de dragage - Résistance à la compression Substitution - Argile

\section{Introduction}

Les quantités de sédiments extraits des ports constituent un véritable problème environnemental. Il est donc important de proposer des solutions pour l'utilisation de ces sédiments en tant que constituant de matériau de construction. Ainsi une étude relative au taux de substitution d'argile dans la composition de briques en incorporant des sédiments de dragage des ports de Tanger et de Larache du nord du Maroc, a été menée.

L'étape de faisabilité technologique de ce type de valorisation a été déjà montrée par des essais préliminaires (BEN ALLAL et al., 2011). Cette étude préliminaire a été approfondie par le présent travail. Des mélanges (argile + sédiments) ont été préparés pour la confection des échantillons de briques. L'optimum de substitution a été déterminé par la mesure des résistances mécaniques sur des briques-éprouvettes. La surface spécifique des mélanges, de l'argile de la briqueterie et des sédiments a été déterminée par la méthode de l'adsorption du bleu de méthylène. La porosité, l'absorption d'eau et la masse volumique apparente des échantillons de briques ont été mesurées. 
La connaissance de la Mer :

un vecteur du développement durable en Méditerranée

\section{Méthodologie}

Une analyse chimique comparative des sédiments des ports de Tanger et Larache (BEN ALLAL et al., 2011) et de l'argile (briqueterie Al Andalous) a été établie. Il ressort de cette étude que la composition chimique des sédiments étudiés avoisine celle de l'argile utilisée par la briqueterie et conforme aux normes internationales (AFNOR, 1983).

\subsection{Préparation des mélanges}

La confection des éprouvettes de briques de forme cylindrique (30 $\mathrm{mm}$ de diamètre et d'un élancement de 3) a été faite pour des mélanges de sédiments et d'argile avec différents taux de substitution : $0 \%, 20 \%, 40 \%$ et $70 \%$ selon la procédure décrite par (SAMARA, 2007). Le sable jouant le rôle de dégraissant (ALVISET, 1994) n’a pas été ajouté au mélange car la teneur en silice dans l'argile de la briqueterie est suffisante. La cuisson des échantillons de briques a été réalisée dans le four industriel de la briqueterie Al Andalous.

\subsection{Mesure de la surface spécifique}

La surface spécifique des sédiments, de l'argile utilisée par la briqueterie Al Andalous ainsi que celle des mélanges, a été déterminée par la méthode d'adsorption du bleu de méthylène. Cette technique permet de mesurer la surface totale externe et interne des particules. Les résultats sont donnés sur la figure 1.

Nous constatons que la surface spécifique des mélanges diminue avec l'ajout des sédiments quel que soit le port d'origine. Les mélanges contribuent à faire chuter la surface spécifique de l'échantillon Témoin. La substitution de l'argile par les sédiments crée donc une augmentation de la taille des particules au sein des mélanges.

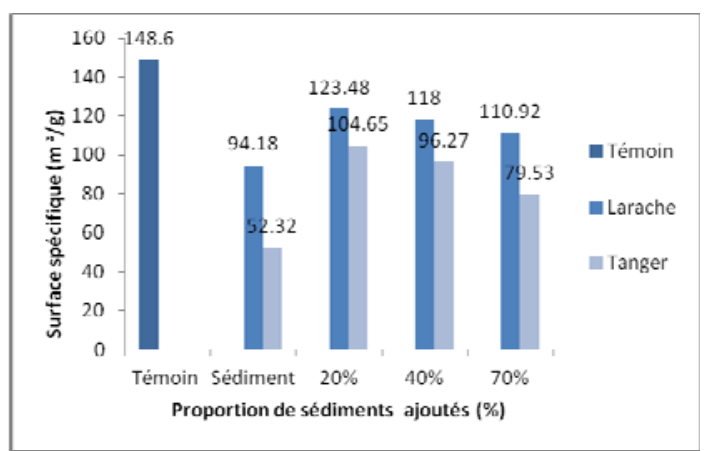

Figure 1.Variation de la surface spécifique des mélanges.

\section{Etude de quelques propriétés des échantillons de briques}

\subsection{Porosité et absorption d'eau}

La porosité est déterminée par le rapport du volume total des vides ouverts du matériau au volume apparent. Elle a une influence importante sur la résistance mécanique, 
l'absorption d'eau et la perméabilité de la brique (HANDISYDE et al., 1976 ; ROSS \& BUTLIN, 1989). Elle a été mesurée sur les briques-éprouvettes par la méthode de la pesée hydrostatique. Le test d'absorption d'eau a été réalisé selon la norme ASTM C6703a (2003). Les valeurs déduites des mesures sont représentées sur la figure 2. La porosité ouverte à l'eau des briques augmente en fonction du taux de substitution ainsi que le coefficient d'absorption d'eau. L'ajout de sédiments augmente par conséquent la taille des pores dans les briques. La porosité est d'autant plus grande pour les briques incorporant le sédiment de Tanger.
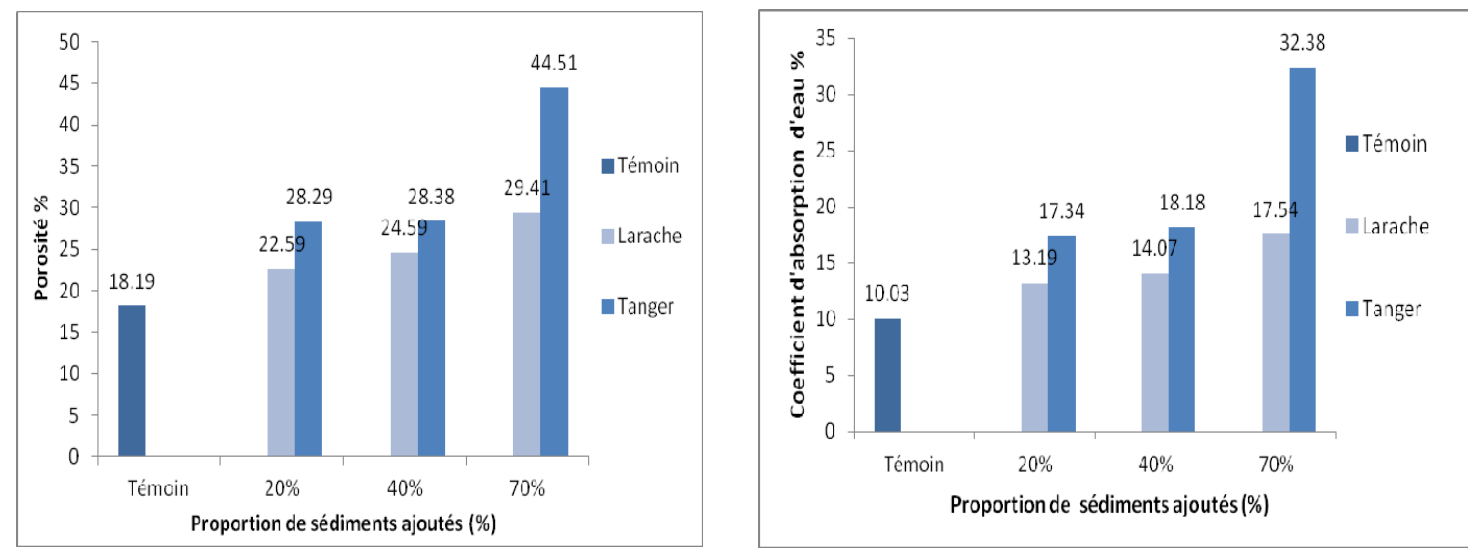

Figure 2. Porosité et coefficient d'absorption d'eau des briques testées.

\subsection{Masse volumique apparente}

Les résultats de la mesure volumique apparente sur les briques-éprouvettes sont donnés sur la figure 3. Nous constatons que la masse volumique apparente des briques diminue avec l'augmentation du taux de substitution de sédiments en bon accord avec les porosités et coefficients d'absorption mesurés. L’incorporation des sédiments rend les briques plus poreuses alors moins denses.

\subsection{Performances mécaniques}

La résistance à la compression, facteur de performance d'une brique, a été déterminée pour différents taux de substitution de sédiments, voir figure 4.

Si l'on observe la figure 4, les valeurs des résistances à la compression montrent que le sédiment de Larache permet de réaliser une brique aux caractéristiques mécaniques voisines de celles du témoin pour un taux de substitution allant jusqu'à $40 \%$. Pour un taux de substitution de $70 \%$ nous obtenons une valeur de 25,76 $\mathrm{MPa}$, soit une diminution de 22\%. La brique renfermant le sédiment de Tanger voit ses caractéristiques mécaniques décroître dès lors que l’on ajoute des sédiments, la meilleure valeur est obtenue pour un ajout de $20 \%$. Au-delà de cette valeur, la résistance diminue. Cette étude montre que la résistance à la compression est influencée par la quantité et la nature du sédiment incorporé dans la brique. Les deux sédiments peuvent 
La connaissance de la Mer :

un vecteur du développement durable en Méditerranée

donc être utilisés comme matière première dans la fabrication des briques, mais à des taux différents et préférentiellement celui de Larache.

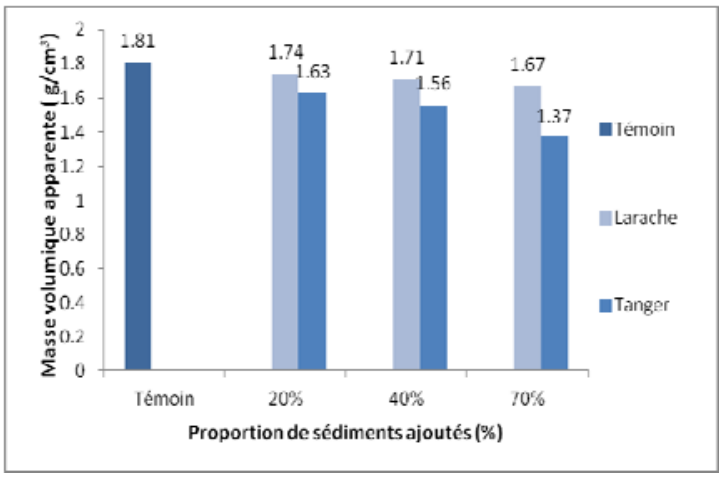

Figure 3. Masse volumique apparente $\left(\mathrm{g} / \mathrm{cm}^{3}\right)$.

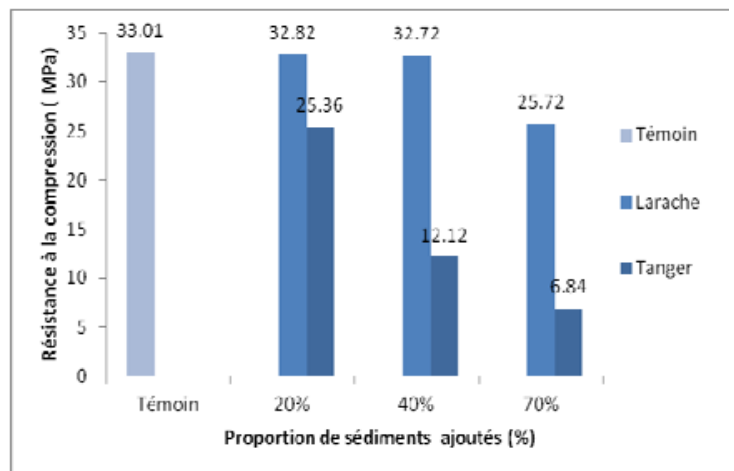

Figure 4.Effet de la substitution des sédiments sur la résistance à la compression.

\section{Conclusion}

Dans cette étude, les résultats obtenus sont très encourageants pour l'utilisation des sédiments de dragage portuaire comme matière première dans la fabrication de briques à l'échelle industrielle mais encore faut-il déterminer les dosages optimaux ? Les briques confectionnées à partir du sédiment du port de Larache à l'échelle du laboratoire présentent des performances mécaniques analogues aux briques de référence jusqu'à un taux de substitution de $40 \%$ ce qui n’est pas le cas pour le sédiment de Tanger.

L’influence d'autres paramètres sur les propriétés inhérentes aux briques reste à étudier, Comme celles relatives à l'humidité, au retrait-gonflement, à l'efflorescence pour établir une fiche technique complète de ces briques élaborées à base de sédiments.

\section{Références bibliographiques}

AFNOR (1983). NF P13-304. Briques pleines ou perforées et blocs perforés en terre cuite. Octobre 1983.

ALVISET L. (1994). Matériaux de terre cuite. Techniques de l’ingénieur. 10 Mai 1994. ASTM C 67-03a (2003). Standard test methods for sampling and testing brick and structural clay tile.

BEN ALLAL L., AMMARI M., FRAR I., AZMANI A., BELMOKHTAR N.E. (2011). Caractérisation et valorisation des sédiments de dragage des ports de Tanger et Larache (Maroc). Revue Paralia, Vol. 4, pp 5.1-5.13. doi :10.5150/revue-paralia.2011.005 HANDISYDE C.C., HASELTINE B.A. (1976). Bricks and brickwork. Rep. No. 142, The Brick Development Association, London.

ROSS K., BUTLIN R.N. (1989).Durability tests for building stone. Building Research Establishment Rep. BR 141, Garston, Watford, U.K.

SAMARA M. (2007). Valorisation des sédiments fluviaux pollués après inertage dans la brique cuite. Thèse de doctorat. Université des Sciences et Techniques de Lille. 